\title{
Somatostatin Receptor Imaging in Patients with Neuroendocrine Tumors: Not Only SPECT?
}

$\mathbf{I}_{\mathrm{n}}$ Medicine, Gabriel et al. (1) report their experience with ${ }^{68} \mathrm{Ga}$-labeled $1,4,7,10$ tetraazacyclododecane- $N, N^{\prime}, N^{\prime \prime}, N^{\prime \prime \prime}$ tetraacetic acid-D-Phe ${ }^{1}-\mathrm{Tyr}^{3}$-octreotide $\left({ }^{68} \mathrm{Ga}\right.$-DOTA-TOC) PET in a series of 84 patients with neuroendocrine tumors (NET). PET was more accurate with a higher detection rate than somatostatin receptor scintigraphy (using ${ }^{111} \mathrm{In}$-DOTA-TOC or ${ }^{99 \mathrm{~m}}$ Tc-labeled hydrazinonicotinyl-Tyr ${ }^{3}$-octreotide [99mTc-HYNIC-TOC]) and CT, providing further clinically relevant information.

NET represent heterogeneous and complex entities derived from neuroendocrine cells, which are characterized

\section{See page 508}

by neurotransmitter, neuromodulator, or neuropeptide hormone production, dense-core secretory granules, and the absence of axons and synapses. Their histopathologic classification has been revised recently (2); in particular, a distinction was made between welldifferentiated NET, which show benign behavior or uncertain malignant potential; well-differentiated neuroendocrine carcinomas, which are characterized by low-grade malignancy; and poorly differentiated neuroendocrine carcinomas of high-grade malignancy. Moreover, NET were also subdivided on the basis of their primary anatomic local-

Received Dec. 27, 2006; revision accepted Jan. 2, 2007.

For correspondence or reprints contact: Orazio Schillaci, Viale G. Mazzini 121, 00195 Rome, Italy. E-mail: orazio.schillaci@uniroma2.it

COPYRIGHT @ 2007 by the Society of Nuclear Medicine, Inc.

DOI: 10.2967/jnumed.106.038653 ization, with the gastrointestinal tract and the lung being the main sites of origin. The simplest clinical classification is between functioning tumors-when a specific clinical syndrome related to hypersecretion of hormones is observedand nonfunctioning tumors-when no specific symptoms related to tumor production are present.

NET are generally considered rare diseases, but their incidence is believed to be higher than reported, primarily due to the relevant number of tumors that go undetected, especially when they are small and clinically silent (3). This aspect was particularly relevant before the introduction of radiolabeled somatostatin analogs for NET imaging, which really represented a major breakthrough in the detection of these neoplasms. In fact, because most NET express a high density of somatostatin receptors, they can be successfully visualized in vivo by somatostatin receptor scintigraphy (SRS). Moreover, the importance of the presence of somatostatin receptors on NET and their management have been established: several studies have shown that somatostatin analogs have antiproliferative effects in normal tissue and NET as well as inhibitory effects on both normal hormone secretion and ectopic release of bioactive products by NET (3).

Today, after more than a decade of experience, SRS, especially with ${ }^{111} \mathrm{In}$ pentetreotide-the most widely used radiolabeled somatostatin analog-has become the main imaging method for evaluating NET overexpression of somatostatin receptors, such as gastroenteropancreatic (GEP) tumors. SRS is routinely performed for localizing the primary tumor, evaluating disease extension, monitoring treatment effects, determining the receptor status as a predictor of response to octreotide therapy, and selecting patients for targeted radionuclide therapy. The results of a European delphi consensus procedure, aimed at establishing guidelines for imaging standardization in NET, clearly indicated that SRS has gained a central role in the diagnosis of these neoplasms, with radiologic procedures such as CT and MRI serving well for completion of the oncologic workup and monitoring of treatment (4). The reported sensitivity of SRS is $80 \%-$ $90 \%$, and it has been found to be superior to other diagnostic imaging methods in identifying and assessing the staging of GEP tumors, except for insulinoma (3). Furthermore, numerous data from the literature indicate that SRS is able to induce a change in the clinical management of a significant proportion (21\%-53\%) of patients with GEP tumors (3). Therefore, performing SRS has become essential to the proper management of patients with different extents of GEP tumors, and it is now recommended as the initial imaging modality in these neoplasms.

Nevertheless, despite this high sensitivity in most NET, the ability of SRS to visualize tumor sites in vivo is closely related to their size and is limited especially in lesions $\leq 1 \mathrm{~cm}$, both primary and metastatic (lymphnodal and hepatic) ones $(3,5)$. To increase the sensitivity of SRS, performing SPECT is mandatory, particularly when tumors are small, located in the abdomen, and not visualized on planar scans, as an overprojection by other tissues or organs (liver, spleen, kidneys, and intestines) that show some variable individual accumulation of the radiopharmaceutical; moreover, SPECT is also very useful in the study of the liver, which always shows ${ }^{111}$ Inpentetreotide uptake (6). The reported 
specificity of SRS is high ( $\sim 90 \%)$, but it can be significantly affected by the physiologic biodistribution of the radiopharmaceutical related to the receptor status of target tissues or to its elimination route via the kidneys and gastrointestinal tract (3). However, the main drawback of SRS is the uncertainty in the anatomic definition, which frequently makes it difficult to precisely localize a focus of abnormal accumulation and identify structures displaying normal activity. These limitations of SRS may be alleviated by combining nuclear medicine with CT or MRI studies; in fact, improved image interpretation has been obtained by fusion of SPECT and CT data, using external (as in the study by Gabriel et al. (1)) or internal markers or using a SPECT/CT hybrid device $(7,8)$.

Because of the superior imaging characteristics of PET, it has been presumed that its use would improve the detection of NET; however, until now, PET has not been routinely used in NET imaging, except in some specialized centers. In fact, ${ }^{18} \mathrm{~F}$ FDG-the only PET tracer that is really widely available-frequently fails to visualize tumors with a low proliferation rate as many NET (9), and high ${ }^{18} \mathrm{~F}$-FDG uptake is observed only in those neoplasms that show an aggressive behavior (10). The development of PET radiopharmaceuticals for detecting NET is of great interest because they can offer increased spatial resolution, when compared with SPECT, and the opportunity to evaluate various aspects of NET biochemistry in vivo; depending on the tracer used, it is possible to selectively measure the function of different metabolic pathways or the expression of enzymes and receptors (11). One of the main advantages of ${ }^{68} \mathrm{Ga}$-DOTATOC for possible routine use in this field is that ${ }^{68} \mathrm{Ga}$ can be produced by an in-house ${ }^{68} \mathrm{Ge} /{ }^{68} \mathrm{Ga}$ generator with a long half-life $(270.8 \mathrm{~d})$. Thus, it can be used for more than a year, and it is also continuously available in nuclear medicine centers without cyclotrons (12). Moreover, as reported by Gabriel et al. (1), DOTA-TOC can be easily and quickly $(<30 \mathrm{~min})$ labeled with ${ }^{68} \mathrm{Ga}$, and synthesis results in a product with high radiochemical purity (13). This ready preparation is an important advantage in comparison with $N^{\alpha}$-(1-deoxy-D-fructosyl)- $N^{\varepsilon}-\left(2-{ }^{18} \mathrm{~F}-\right.$ fluoropropionyl)-Lys ${ }^{0}-\mathrm{Tyr}^{3}$-octreotate (Gluc-Lys $\left.\left({ }^{18} \mathrm{~F}-\mathrm{FP}\right)-\mathrm{TOCA}\right)$, a glycosylated ${ }^{18} \mathrm{~F}$-labeled somatostatin analog recently proposed for PET of NET, which requires a multistep synthesis with an overall preparation time of about $3 \mathrm{~h}$ and has limited radiochemical yield (14).

Preclinical data indicated that ${ }^{68} \mathrm{Ga}$ DOTA-TOC shows a very high affinity for somatostatin receptors 2 and 5 (11). The preliminary clinical results of Hofmann et al. (15), in 8 patients with histologically proven metastatic NET, demonstrated that this PET tracer, within a period of 30-40 min after injection, achieves higher tumor-tonontumor ratios than SRS. ${ }^{68} \mathrm{Ga}$-DOTATOC identified all previously known lesions, whereas ${ }^{111}$ In-pentetreotide scintigraphy identified only $85 \%$, and furthermore detected additional small lesions, including brain metastases. Other important differences in comparison with conventional SRS are the lower kidney accumulation of ${ }^{68} \mathrm{Ga}$ DOTA-TOC and the visualization of small organs with physiologic somatostatin receptors. These findings have been then confirmed in a small group of 4 patients with NET, in which this PET tracer was superior to ${ }^{111}$ In-pentetreotide, especially in visualizing small tumors or lesions bearing only a low density of somatostatin receptors (16).

After these previous reports on a very limited number of patients, the article by Gabriel et al. (1) confirms the feasibility and the high accuracy of ${ }^{68} \mathrm{Ga}$-DOTA-TOC PET as a promising method for visualizing NET in a larger patient population. On a patient basis, PET accuracy (96\%) was significantly higher than that of $\mathrm{CT}(75 \%)$ and SPECT $(58 \%)$; in particular, ${ }^{68} \mathrm{Ga}-$ DOTA-TOC imaging results were truepositive in 32 patients whose SPECT findings were false-negative, and it was able to visualize more lesions $(n=$
375) than SPECT $(n=302)$ and CT $(n=295)$. Furthermore, PET detected more tumor sites than scintigraphy in lymph nodes, in the liver and in the bone, thus allowing an accurate staging that is useful for proper patient management. These results provided further clinically relevant information in $14 \%$ of the patients compared with SPECT and in $21 \%$ compared with CT. The capability of influencing treatment is of the utmost importance for a diagnostic method when examining NET patients, in whom-after the diagnosis is established-correct localization of the primary tumor and evaluation of the extent of the disease is essential to determine whether curative surgical resection, the most effective therapy, is possible.

It is worth noting that ${ }^{68} \mathrm{Ga}$-DOTATOC uptake was not influenced by the functionality of the tumor; in fact, the broad majority of patients (i.e., 57/ 84) had a nonsecreting neoplasm. This finding is significant, because ${ }^{18} \mathrm{~F}-$ labeled 3,4-dihydroxyphenylalanine and ${ }^{11} \mathrm{C}$-labeled 5-hydroxytryptophan-2 promising PET radiopharmaceuticals for NET imaging that accumulate via metabolic mechanisms - seem to have a limitation in detecting nonfunctioning tumors $(17,18)$. Moreover, both of these tracers are not able to provide information about the somatostatin receptor status - that is fundamental for treatment options. On the other hand, a recent article evaluated the pharmacokinetics of ${ }^{68} \mathrm{Ga}$-DOTA-TOC in 22 patients with metastatic NET scheduled for ${ }^{90}$ Y-DOTA-TOC therapy (19) and revealed that tumor uptake of this tracer is influenced primarily by high receptor binding. The metastatic lesions expressing somatostatin receptors were visualized with a high mean global standardized uptake value (i.e., 8.73). Further studies are required to define the potential clinical role of quantitative ${ }^{68} \mathrm{Ga}-\mathrm{DOTA}-\mathrm{TOC}$ in accurately selecting patients for radionuclide therapy and in assessing the therapeutic outcome.

As clearly highlighted in the article by Gabriel et al. (1), the very specific accumulation of ${ }^{68} \mathrm{Ga}$-DOTA-TOC 
may represent a limitation, because the anatomic delineation of abnormal findings is not easy without image fusion and because it may cause overinterpretation. The systematic use of PET/CT can solve the first potential problem in image analysis, which, however, should be done carefully to avoid false-positive results in organs with physiologic uptake, such as the pancreatic head.

In conclusion, the results of the study by Gabriel et al. (1) suggest that somatostatin receptor imaging using ${ }^{68}$ Ga-DOTA-TOC PET is a very promising tool for evaluating patients with NET, showing a high diagnostic accuracy, superior to conventional SRS. PET offers better resolution than SPECT and enables quantitative assessments of radioactivity in different tissues over time, which can be used for radionuclide therapy dosimetry, and a shorter duration of scan, which is completed within $2 \mathrm{~h}$ from the radiopharmaceutical injection. It is likely that this tracer will be routinely used in the near future: this will depend primarily on its easy worldwide availability and the capillary diffusion of PET/CT. However, it is necessary to collect further data confirming the clinical usefulness of ${ }^{68} \mathrm{Ga}$-DOTATOC PET before it can replace SRS in the everyday nuclear medicine practice.
Orazio Schillaci

University Tor Vergata

Rome, Italy

\section{REFERENCES}

1. Gabriel M, Decristoforo C, Kendler D, et al. ${ }^{68} \mathrm{Ga}-$ DOTA-Tyr ${ }^{3}$-octreotide PET in neuroendocrine tumors: comparison with somatostatin receptor scintigraphy and CT. J Nucl Med. 2007;48:508-518.

2. Solcia E, Klöppel G, Sobin LH. Histological typing of endocrine tumours. In: Solcia E, Klöppel $\mathrm{G}$, Sobin LH, eds. International Histological Classification of Tumours. 2nd ed. Berlin, Germany: Springer-Verlag; 2000:61-68.

3. Gibril F, Jensen RT. Diagnostic uses of radiolabelled somatostatin receptor analogues in gastroenteropancreatic endocrine tumours. Dig Liver Dis. 2004;36(suppl 1):S106-S120.

4. Ricke J, Klose KJ, Mignon M, Oberg K, Wiedenmann B. Standardisation of imaging in neuroendocrine tumours: results of a European delphi process. Eur J Radiol. 2001;37:8-17.

5. Dromain C, de Baere T, Lumbroso J, et al. Detection of liver metastases from endocrine tumors: a prospective comparison of somatostatin receptor scintigraphy, computed tomography, and magnetic resonance imaging. $J$ Clin Oncol. 2005;23: 70-78.

6. Schillaci O, Scopinaro F, Angeletti S, et al. SPECT improves accuracy of somatostatin receptor scintigraphy in abdominal carcinoid tumors. $\mathrm{J} \mathrm{Nucl}$ Med. 1996;37:1452-1456.

7. Schillaci O. Functional-anatomical image fusion in neuroendocrine tumors. Cancer Biother Radiopharm. 2004;19:129-134.

8. Krausz Y, Israel O. Single-photon emission computed tomography/computed tomography in endocrinology. Semin Nucl Med. 2006;36: 267-274.

9. Adams S, Baum R, Rink T, et al. Limited value of fluorine-18 fluorodeoxyglucose positron emission tomography for the imaging of neuroendocrine tumours. Eur J Nucl Med. 1998;25:79-83.
10. Pasquali C, Rubello D, Sperti C, et al. Neuroendocrine tumor imaging: Can ${ }^{18} \mathrm{~F}$-fluorodeoxyglucose positron emission tomography detect tumors with poor prognosis and aggressive behavior? World J Surg. 1998;22:588-592.

11. Rufini V, Calcagni ML, Baum RP. Imaging of neuroendocrine tumors. Semin Nucl Med. 2006;36: 228-247.

12. Maecke HR, Hofmann M, Haberkorn U. ${ }^{68} \mathrm{Ga}-$ Labeled peptides in tumor imaging. $\mathrm{J} \mathrm{Nucl} \mathrm{Med}$. 2005;46(suppl 1):172S-178S.

13. Breeman WA, de Jong M, de Blois E, Bernard BF, Konijnenberg M, Krenning EP. Radiolabelling DOTA-peptides with ${ }^{68} \mathrm{Ga}$. Eur J Nucl Med Mol Imaging. 2005;32:478-485.

14. Meisetschlager G, Poethko T, Stahl A, et al. GlucLys $\left(\left[{ }^{18} \mathrm{~F}\right] \mathrm{FP}\right)-\mathrm{TOCA}$ PET in patients with SSTRpositive tumors: biodistribution and diagnostic evaluation compared with [ $\left.{ }^{111} \mathrm{In}\right] \mathrm{DTPA}-\mathrm{octreotide.}$ J Nucl Med. 2006;47:566-573.

15. Hofmann M, Maecke H, Börner AR, et al. Biokinetics and imaging with the somatostatin receptor PET radioligand ${ }^{68} \mathrm{Ga}$-DOTATOC: preliminary data. Eur J Nucl Med. 2001;28:17511757.

16. Kowalski J, Henze M, Schumacher J, Macke HR, Hofmann M, Haberkorn U. Evaluation of positron emission tomography imaging using $\left[{ }^{68} \mathrm{Ga}\right]-$ DOTA-D $\mathrm{Phe}^{1}$-Tyr ${ }^{3}$-octreotide in comparison to [ $\left.{ }^{111} \mathrm{In}\right]-D T P A O C$ SPECT: first results in patients with neuroendocrine tumors. Mol Imaging Biol. 2003;5:42-48.

17. Eriksson B, Orlefors H, Oberg K, Sundin A, Bergstrom M, Langstrom B. Developments in PET for the detection of endocrine tumours. Best Pract Res Clin Endocrinol Metab. 2005;19:311-324.

18. Sundin A, Eriksson B, Bergstrom M, Langstrom B, Oberg K, Orlefors H. PET in the diagnosis of neuroendocrine tumors. Ann N Y Acad Sci. 2004; 1014:246-257.

19. Koukouraki S, Strauss LG, Georgoulias V, et al. Evaluation of the pharmacokinetics of ${ }^{68} \mathrm{Ga}-68$ DOTATOC in patients with metastatic neuroendocrine tumours scheduled for ${ }^{90} \mathrm{Y}$-DOTATOC therapy. Eur J Nucl Med Mol Imaging. 2006;33:460-466. 